\title{
Validity and reliability of the Persian version of the multiple sclerosis quality of life questionnaire
}

\author{
H. Ghaem, A. Borhani Haghighi, P. Jafari, A. R. Nikseresht \\ Department of Epidemiology, School of Health, Shiraz, Iran
}

\begin{abstract}
Background and Aims: To translate and test the reliability and validity of the Multiple Sclerosis Quality of Life Questionnaire (MSQoL-54) in Iranian MS patients. Setting and Design: Using a standard "forward-backward" translation, cognitive debriefing and cultural adaptation procedure, the English version of the MSQoL-54 was translated to Persian which is the Iranian official language. Materials and Methods: The subjects were multiple sclerosis (MS) patients referred to Motaharri clinic, Shiraz, South of Iran. Demographic data were recorded. Epidemiological data concerning MS type, duration of the disease, Functional System Score (FSS) and Expanded Disability Status Scale (EDSS) of patients were also provided by a qualified neurologist. Statistical Analysis: The reliability of the questionnaire was assessed by Cronbach's alpha coefficient. Construct validity was assessed through factor analysis. Factor analysis was performed to determine that the Persian version is a two-dimensional measure including physical and mental parameters. Results: Multiple sclerosis patients (female:106 (75.2\%), male:35 (24.8\%)), with a mean $\pm S D$ age of $32.2 \pm 9.8$ years were enrolled in the study. Cronbach's $\alpha$ was 0.962 . There were no significant differences between each item and the mean of physical and mental scores of MSQoL-54, regarding sex, marital status and education. There was a negative significant correlation between EDSS and physical health, role limitation due to physical problems, pain, energy, health perception, social function, cognitive function, health distress, overall Quality of Life. The scaling success rates were $100 \%$, demonstrating convergent validity of each scale. Factor analysis confirmed the construct validity of the questionnaire. Conclusions: The Persian version of the MSQoL-54 questionnaire has a good structural characteristic, it is a reliable and valid instrument and can be used for measuring the effect of MS on the Quality of Life.
\end{abstract}

Key words: MSQoL-54, multiple sclerosis, reliability, validity
Multiple sclerosis (MS) is the most common nontraumatic cause of disability in the world. Multiple sclerosis is an inflammatory demyelinating disorder of the Central Nervous System (CNS) that affects individuals in their most productive ages and is a tremendous burden for years to come. ${ }^{[1]}$ Physical impairments and psychological changes can influence self-esteem and Quality of Life in MS patients. ${ }^{[2]}$

Over the past decade Health-Related Quality of Life (HRQoL) instruments have become increasingly popular as end point tools in clinical studies for measuring patient-assessed health status. ${ }^{[3,4]}$ While the effect of MS on life expectancy remains controversial, the disease's negative effect on HRQoL is documented and a topic currently undergoing clinical study. ${ }^{[5,6]}$ Also, several studies have shown that HRQoL assessments provide unique information on impairment for MS patients. ${ }^{[7-9]}$ As an alternative indicator of the impact of the disease on a patient's life, self-related HRQoL focuses more attention on MS patients as a whole, in addition to focusing on physical problems ${ }^{[10]}$ and when the goal of treatment is to improve patients' well-being rather than to increase survival, as in chronic conditions, HRQoL measurements are essential. ${ }^{[11]}$

There has been an upsurge in interest in HRQoL in the medical literature over the past few years. Of the 100 or so papers examining Quality of Life and Multiple Sclerosis, 80\% have been published since 1991. ${ }^{[12]}$

Health-Related Quality of Life instruments are expected to be of particular value in the routine care of people with MS, where they may facilitate the detection of disease aspects that would otherwise go unrecognized and help clinicians appreciate patient priorities, particularly in terms of treatment goals. These instruments facilitate physician-patient communication and promote shared decision-making. However, it appears that they are little used routine clinical approaches to people with MS. ${ }^{[13]}$ 
The ultimate aim of measuring HRQoL is to provide a comprehensive assessment of patients' health status, to serve as a baseline to tailor pharmacological interventions and assess their effectiveness, both in the clinical trial setting and in routine care. In addition, HRQoL data from clinical trials can provide information that clinicians can use to discuss with their patients. ${ }^{[13,14]}$

A few available studies have employed generic HRQoL inventories in MS patients and only recently have disease-specific instruments begun to be used. ${ }^{[15-19]}$ The SF-36 was supplemented by 18 additional items (MS-18 module) to obtain the MS Quality of Life 54 (MSQoL-54) questionnaire, specific for MS patients. ${ }^{[15]}$ The MSQoL54 has been translated into several languages..$^{[5,11,20-22]}$

There are approximately 25000 MS patients in Iran. Therefore, we need to have a valid and reproductive instrument to assess the quality of life among Iranian patients. The objective of this study was to assess the validity and reliability of the MSQoL-54, to make it available to the Iranian scientific community for clinical research and practice.

\section{Materials and Methods}

\section{Instrument}

MSQoL-54

The SF-36 questionnaire is one of the most widely used HRQoL instruments in the United States. ${ }^{[1]]}$ It was devised to satisfy the minimum psychometric standards necessary for group comparisons involving general health dimensions (not specific to age, disease or treatment group). ${ }^{[14,23]}$ The SF-36 measures two major health concepts by means of two composite scores (Physical Health Composite and Mental Health Composite) obtained from eight multi-item scales: physical function; role limitation-physical; bodily pain; general health; vitality; social function; role limitationemotional; and mental health; in all 36 items. The composite instrument composed of SF-36 and MS18, is MSQoL-54 which contains 52 items grouped into 12 scales, plus two individual items (satisfaction with sexual function and change in health). ${ }^{[15]}$ Sum of subtotals; physical function, health perceptions, energy/fatigue, role limitation-physical, pain, sexual function, social function, health distress makes the physical health composite and sum of subtotals; health distress, overall quality of life, emotional well-being, role limitation-emotional, cognitive function makes the mental health composite.

An additional question is also presented which asks about self-evaluated change in health status. The MS-18 module originally devised in the United States in $1995^{[24]}$ adds 18 additional items to SF-36 concerned with the following areas: health distress; sexual function; satisfaction with sexual function; overall quality of life; cognitive function; and energy.

\section{Pittsburgh sleep quality index and fatigue severity scale}

The Pittsburgh Sleep Quality Index (PSQI), is an effective 18-item self-report instrument used to measure the quality and patterns of sleep in older adults. It differentiates "poor" from "good" sleep by measuring seven areas: subjective sleep quality, sleep latency, sleep duration, habitual sleep efficiency, sleep disturbances, use of sleeping medication and daytime dysfunction over the last month. ${ }^{[25]}$

\section{The fatigue severity scale (FSS)}

This is a method of evaluating fatigue in multiple sclerosis. It is designed to differentiate fatigue from clinical depression, since both share some of the same symptoms. ${ }^{[15]}$

\section{Expanded disability status scale (EDSS)}

Interest in measuring outcomes in MS has increased markedly over the past 20 years. Standardized instruments have been developed and the most widelyused one is the expanded disability status scale (EDSS) ${ }^{[7]}$ which is a mixed impairment/activity limitations scale based on the neurological examination of eight functional systems, plus ambulation/mobility status. Despite major limitations-bias towards locomotor function, variable sensitivity to change according to scale score and suboptimal inter-rater reliability, the EDSS is increasingly being used by researchers and clinicians because its scores are readily understood by all. The EDSS scale extends from 0 (normal neurological examination) to 10 (death from MS) in 0.5 unit increments. ${ }^{[7]}$

\section{Translation and adaptation}

The standard "forward-backward" procedure ${ }^{[26]}$ was applied to translate the questionnaire from English to Persian. Two independent bilinguals translated the 54 items into Persian and then the preliminary version was back translated into English.

The aim of cultural adaptation was to produce a version that was conceptually as close as possible to the original questionnaire, considering the patients' understanding. The Persian version of MSQoL-54 was administered to 20 MS patients referred to the Department of Neurology, Motahari clinic, Shiraz, Iran.

The patients were asked to make a note beside the unclear or vague questions. The results of pilot testing and related interviews were summarized. The problematic items were changed according to the pilot results and patients' comments.

Subsequently, cultural adaptation (for instance, games like bowling and golf are not common and widely known in Iran and so culturally adapted games which could replace them were two activities like "vasati" (the middle one) and "haft-sang" (seven stones), and due to religious 
beliefs and cultural habits, the questions concerning sexual function were answered only by married patients and "Partner" was translated to "Wife" and "Husband") was provided.

Demographic data like age, sex, marital status, socioeconomic status and educational level were recorded. Epidemiological data concerning MS type, duration of the disease, FSS and EDSS of patients were also provided by a qualified neurologist.

\section{Sample size}

It has been suggested that the adequate sample size for validation of a quality of life questionnaire is between $100-400 .{ }^{[27]}$

\section{Patients}

The subjects were regular patients (both newly diagnosed and follow-up ones) who were referred to the MS clinic at Nemazee Hospital in Shiraz, from June to December 2005.

Inclusion criteria were clinically definite or laboratory supported MS according to Poser criteria. ${ }^{[28]}$

All the patients signed the informed consent and also this study was approved by the ethics committee (NO: 84-2526).

The literate patients filled out the questionnaire by themselves. For illiterate patients the questionnaire was filled out by verbal communication with unbiased test operators.

Then the patient was asked two further questions, regarding the relevance and clarity of the questions. The time which was taken by each patient to complete the questionnaire was recorded.

After filling out the questionnaire, the coordinator checked the questions for missing items, for example, if one or more questions were not answered, she asked the patient to complete them. Therefore, no missing data existed in the questionnaire (except for sexual function questions, since they were answered only by married patients).

\section{Statistical analysis}

The MSQoL-54 scale scores were computed using the Likert method for summed ratings and the raw scores were linearly transformed into 0-100 scales: the higher the transformed score, the better the patient's HRQoL. ${ }^{[29]}$ The patient acceptability was assessed from the mean period of time required to complete the questionnaire. In addition, the response consistency index was applied to calculate the percentage of logical inconsistencies committed in completing the questionnaire, that analyzed the responses using internal checks based on answers to 15 pairs of items. For example, being able "to walk a kilometer" but "not a hundred meters" is considered as an inconsistency in scoring the response consistency index. ${ }^{[14,29]}$ Grouping and scaling assumptions were assessed using standard psychometric instruments described in the papers that presented the original questionnaire. ${ }^{[14,29]}$ Briefly, as the questionnaire is based on a multidimensional conceptualization of health, the multitrait analysis approach was adopted to test whether conceptualization into domains fitted the data and whether the results of the Persian questionnaire replicated the results obtained with the English language questionnaire in terms of convergence.

\section{Validity and reliability}

Internal consistency reliability was computed by Cronbach's alpha (recommended value $\alpha \geq 0.70$ ). ${ }^{[30,31]}$ Spearman correlation coefficient was used to assess convergency. Construct validity was assessed through Factor Analysis. Factor Analysis is a statistical technique used to explain variability among observed random variables in terms of fewer unobserved random variables called factors. ${ }^{[27]}$ Clinical validity was assessed comparing mean MSQOL-54 scores by patient's age, severity of MS symptoms over the preceding year, EDSS score, FSS, PSQI, disease duration, education, marital status. The Mann-Whitney U test was employed for these comparisons. The statistical software program SPSS 13 was used.

\section{Results}

Multiple sclerosis patients aged 16-60 years filled out the questionnaire. The mean age of the patients was 32.6 \pm 9.6 years (male: $33.4 \pm 10$ and female: $31.7 \pm 9.6$ ). From 141 MS patients $24.8 \%$ were male (35) and $75.2 \%$ were female (106). The mean age at diagnosis was $28.9 \pm 8.8$ years. The mean duration from diagnosis was $3.8 \pm 7.2$ years. Table 1 shows the patients' characteristics.

Regarding the type of MS, there were 105 (74.5\%) relapsing-remitting, four $(2.8 \%)$ primary progressive, 28 (19.9\%) secondary progressive and four (2.8\%) relapsing progressive.

The number of patients who had abnormalities in a particular functional system was 86(61\%) pyramidal, 37 (26.2\%) brainstem, 47 (33.3\%) cerebellar, 84 (59.6) sensory, 30 (21.3\%) bowel, 30 (21.3\%) cerebral, 60 (46.8\%) visual and 20 (14.2\%) others (spinal, motor, sexual).

The mean \pm SD EDSS was $2.3 \pm 2.1$. From $141 \mathrm{MS}$ patients 82 (58.1\%) had EDSS $\leq 2,36$ (25.5\%) EDSS 2.5-4.5, and 23 EDSS $\geq 5$.

From the above-mentioned MS patients $4.2 \%$ were illiterate (six) and 95.8\% were literate (135). Completion of the questionnaire took an average of $19 \mathrm{~min}$ (illiterate patients $17 \mathrm{~min}$. vs. literate patients $21 \mathrm{~min}$ ).

The reliability of whole 54 questions of the questionnaire was obtained by Cronbach's alpha coefficient $(\alpha=0.962)$. Cronbach's alpha coefficient of whole 54 questions of the questionnaire according to age, sex, marital status, 
Table 1: Patients' characteristics and descriptive clinical data

\section{Variables}

EDSS, Mean \pm SD

(Range)

Median

Age, Mean \pm SD

(Range)

Years of education,

Mean \pm SD

(Range)

Age at diagnosis

Mean \pm SD

(Range)

Duration from diagnosis

Mean \pm SD

(Range)

Fatigue Mean \pm SD

(Range)

Median

Gender

Male

Female

Marital status

Married

Single

MS type

relapsing-remitting

primary progressive

secondary progressive

relapsing progressive

EDSS

$\leq 2$

2.5-4.5

$\geq 5$

$$
\begin{gathered}
2.3 \pm 2.1 \\
0-9 \\
2 \\
32.6 \pm 9.6 \\
16-60 \\
11.25 \pm 4.47 \\
0-19 \\
\\
28.9 \pm 8.8 \\
16-49 \\
3.8 \pm 7.2 \\
0-21 \\
31.98 \pm 12.89 \\
7-49 \\
34 \\
\mathrm{~N}(\%) \\
35(24.8) \\
106(75.2) \\
88(62.5) \\
53(37.5) \\
105(74.5) \\
4(2.8) \\
28(19.9) \\
4(2.8) \\
82(58.1) \\
36(25.5) \\
23(16.3) \\
\\
\\
\\
\end{gathered}
$$

$\mathrm{SD}=$ Standard deviation, EDSS: Expanded disability status scale

education, Kurtzke's EDSS, and duration of disease was acceptable [Table 2].

Spearman correlation used for assessing convergent validity - between items within scales and between-scale

\begin{tabular}{|c|c|c|c|c|c|}
\hline Scale & $\begin{array}{l}\text { No. of items } \\
\text { per scale }\end{array}$ & $\begin{array}{l}\text { Convergent validity } \\
\text { (range of correlation) }\end{array}$ & $\begin{array}{l}\text { Scaling } \\
\text { success }^{1}\end{array}$ & $\begin{array}{c}\text { Scaling success } \\
\text { rate }^{2}\end{array}$ & $\begin{array}{c}\text { Internal consistency } \\
(\text { Cronbach's } \alpha)\end{array}$ \\
\hline Physical health & 10 & $0.675-0.848$ & $10 / 10$ & 100 & 0.941 \\
\hline $\begin{array}{l}\text { Role limitation due to physical } \\
\text { problems }\end{array}$ & 4 & $0.777-0.864$ & $4 / 4$ & 100 & 0.851 \\
\hline $\begin{array}{l}\text { Role limitation due to } \\
\text { emotional problems }\end{array}$ & 3 & $0.830-0.871$ & $3 / 3$ & 100 & 0.814 \\
\hline Pain & 3 & $0.862-0.889$ & $3 / 3$ & 100 & 0.854 \\
\hline Emotional well-being & 5 & $0.732-0.811$ & $5 / 5$ & 100 & 0.822 \\
\hline Energy & 5 & $0.618-0.805$ & $5 / 5$ & 100 & 0.774 \\
\hline Health perception & 5 & $0.476-0.798$ & $5 / 5$ & 100 & 0.696 \\
\hline Social function & 3 & $0.709-0.804$ & $3 / 3$ & 100 & 0.654 \\
\hline Cognitive function & 4 & $0.792-0.923$ & $4 / 4$ & 100 & 0.916 \\
\hline Health distress & 4 & $0.813-0.913$ & $4 / 4$ & 100 & 0.896 \\
\hline Sexual function & 4 & $0.781-0.928$ & $4 / 4$ & 100 & 0.914 \\
\hline Overall quality of life & 2 & $0.795-0.942$ & $2 / 2$ & 100 & 0.892 \\
\hline
\end{tabular}
correlations and for all scales were acceptable [Table 3]. The scaling success rates were $100 \%$ for the convergent validity of each scale [Table 3].

There were no statistically significant differences between each item and the mean physical and mental
Table 2: Internal consistency of MSQoL-54 by age, gender, marital status, education, expanded disability status scale and duration of disease

\begin{tabular}{lcc}
\hline Variable & N & Cronbach's coefficient \\
Age (year) & & \\
$16-30$ & 70 & 0.955 \\
$31-40$ & 39 & 0.964 \\
$41-60$ & 32 & 0.952 \\
Gender & & \\
$\quad$ Male & 35 & 0.972 \\
Female & 106 & 0.957 \\
Marital status & & \\
Single & 53 & 0.981 \\
Married & 88 & 0.962 \\
Years of education & & \\
$0-11$ & 51 & 0.964 \\
$12-15$ & 57 & 0.963 \\
$16+$ & 33 & 0.951 \\
Expanded disability status scale & & \\
$0-2$ & 82 & 0.953 \\
$2.5-4.5$ & 36 & 0.934 \\
$5-9$ & 23 & 0.941 \\
Duration of disease (year) & & \\
$0-1$ & 73 & 0.952 \\
$2-4$ & 35 & 0.979 \\
$\geq 5$ & 33 & 0.954 \\
\hline
\end{tabular}

scores of MSQoL-54 of males versus females (physical: $P=0.310$, mental: $P=0.642$ ) and married versus single cases (physical: $P=0.344$, mental: $P=0.266$ ). Table 4 shows the results of the internal consistency of each subscale of MSQoL-54 regarding gender and marital status, respectively. Table 5 shows the results of internal consistency of each subscale of MSQoL-54 according to EDSS and MS type.

Mann-Whitney U test showed that the physical health, role limitation due to physical problems, energy and health perception scales have a strong association with marital status (single patients' scores were greater than married patients' ones) [Table 6].

There was a negative significant correlation between EDSS and physical health $(r=-0.429, P<0.001)$, role limitation due to physical problems $(r=-0.243$,

1: Number of correlations between items and hypothesized scale corrected for overlap $>0.4$ / total number of convergent validity tests. 2 : scaling success rate is a previous column as percentage 
Table 4: Internal consistency (Cronbach's $\alpha$ ) of each subscale of MSQoL-54 by gender and marital status

\begin{tabular}{|c|c|c|c|c|}
\hline Scale & Male (Cronbach's $\alpha$ ) & Female (Cronbach's $\alpha$ ) & Single (Cronbach's $\alpha$ ) & Married (Cronbach's $\alpha$ ) \\
\hline Physical health & 0.946 & 0.939 & 0.914 & 0.945 \\
\hline $\begin{array}{l}\text { Role limitation due to physical } \\
\text { problems }\end{array}$ & 0.869 & 0.843 & 0.801 & 0.863 \\
\hline $\begin{array}{l}\text { Role limitation due to } \\
\text { emotional problems }\end{array}$ & 0.898 & 0.781 & 0.769 & 0.826 \\
\hline Pain & 0.868 & 0.836 & 0.838 & 0.873 \\
\hline Emotional well-being & 0.782 & 0.828 & 0.871 & 0.788 \\
\hline Energy & 0.879 & 0.702 & 0.772 & 0.746 \\
\hline Health perception & 0.673 & 0.690 & 0.764 & 0.591 \\
\hline Social function & 0.702 & 0.597 & 0.650 & 0.597 \\
\hline Cognitive function & 0.947 & 0.892 & 0.916 & 0.921 \\
\hline Health distress & 0.885 & 0.896 & 0.904 & 0.885 \\
\hline Sexual function & 0.950 & 0.879 & 0.855 & 0.914 \\
\hline Overall quality of life & 0.915 & 0.877 & 0.931 & 0.855 \\
\hline
\end{tabular}

Table 5: Internal consistency (Cronbach's $\alpha$ ) of each subscale of MSQoL-54 by expanded disability status scale and MS type

\begin{tabular}{|c|c|c|c|c|c|c|c|}
\hline \multirow[b]{2}{*}{ Scale } & \multicolumn{3}{|c|}{ Expanded disability status scale } & \multicolumn{4}{|c|}{ Multiple sclerosis type } \\
\hline & $0-2$ & $2.5-4.5$ & $5-9$ & RR & PP & SP & RP \\
\hline Physical health & 0.913 & 0.914 & 0.968 & 0.925 & 0.988 & 0.964 & 0.823 \\
\hline Role limitation due to physical problems & 0.875 & 0.819 & 0.929 & 0.837 & 1.00 & 0.892 & 0.889 \\
\hline Role limitation due to emotional problems & 0.797 & 0.781 & 0.930 & 0.787 & 1.00 & 0.888 & 0.750 \\
\hline Pain & 0.805 & 0.869 & 0.841 & 0.837 & 0.932 & 0.860 & 0.680 \\
\hline Emotional well-being & 0.810 & 0.731 & 0.842 & 0.793 & 0.779 & 0.894 & 0.957 \\
\hline Energy & 0.793 & 0.697 & 0.705 & 0.787 & 0.900 & 0.795 & 0.800 \\
\hline Health perception & 0.748 & 0.727 & 0.920 & 0.727 & 0.972 & 0.733 & 0.781 \\
\hline Social function & 0.702 & 0.735 & 0.723 & 0.750 & 0.840 & 0.744 & 0.720 \\
\hline Cognitive function & 0.879 & 0.702 & 0.900 & 0.919 & 0.762 & 0.938 & 0.815 \\
\hline Health distres & 0.904 & 0.871 & 0.825 & 0.879 & 0.733 & 0.899 & 0.853 \\
\hline Sexual function & 0.824 & 0.957 & 0.915 & 0.891 & 0.929 & 0.702 & 0.776 \\
\hline Overall quality of life & 0.884 & 0.844 & 0.914 & 0.874 & 0.874 & 0.881 & 0.992 \\
\hline
\end{tabular}

RR: Relapsing-remitting, PP: Primary progressive, SP: Secondary progressive, RP: Relapsing progressive

Table 6: Significant association between subscale of MSQoL-54 and marital status

\begin{tabular}{|c|c|c|c|c|}
\hline Scale & Single $=53$ Mean \pm SD & Married = 88 Mean \pm SD & $\mathbf{Z}$ & $P$-value \\
\hline Physical health & $68.9 \pm 26.7$ & $50.2 \pm 32.9$ & -3.315 & 0.001 \\
\hline Role limitation due to physical problems & $47.2 \pm 39.4$ & $34.7 \pm 40.4$ & -2.151 & 0.031 \\
\hline Energy & $48.4 \pm 20.1$ & $39.3 \pm 19.9$ & -2.872 & 0.004 \\
\hline Health perception & $56.1 \pm 23.1$ & $47.4 \pm 20.8$ & -2.190 & 0.029 \\
\hline
\end{tabular}

RR: Relapsing-remitting, PP: Primary progressive, SP: Secondary progressive, RP: Relapsing progressive

$P=0.011)$, pain $(r=-0.216, P=0.025)$, energy $(r=-0.280$, $\mathrm{P}=0.003)$, health perception $(r=-0.372, \mathrm{P}<0.001)$, social function $(\mathrm{r}=-0.424, \mathrm{P}<0.001)$, cognitive function $(\mathrm{r}=-$ $0.204, P=0.035)$, health distress $(r=-0.241, P=0.012)$, overall quality of life $(r=-0.288, P=0.003)$.

There was a positive significant correlation between years of education and physical health $(\mathrm{r}=0.311$, $\mathrm{P}<0.001$ ), role limitation due to physical problems $(\mathrm{r}=0.244, \mathrm{P}=0.004)$, pain $(\mathrm{r}=0.249, \mathrm{P}=0.003)$, overall quality of life $(r=0.434, P=0.016)$.

Between gender and each scale there was no significant association.

There was a negative correlation between patients' age and physical health $(r=-0.398, P<0.001)$, role limitation due to physical problems $(r=-0.253, P=0.003)$, pain $(r=-0.272, P<0.001)$, energy $(r=-0.323, P<0.001)$, health perception $(r=-0.250, P=0.003)$, social function $(r=-0.315, P<0.001)$, overall quality of life $(r=-0.197$, $P=0.020)$.

There was a negative correlation between fatigue score and physical health composite $(r=-0.679, \mathrm{P}<0.001)$ and mental health composite $(r=-0.811, P<0.001)$ and also between sleep score and physical health composite $(\mathrm{r}=-0.486, \mathrm{P}<0.001)$ and mental health composite $(\mathrm{r}=$ $0.543, \mathrm{P}<0.001)$.

Factor analysis was performed to determine whether the Persian version is a two-dimensional measure, including physical and mental health composite parameters [Table 7]. In our result Pain and Energy/Fatigue are correlated to the Mental Health Composite. The bold number in the table shows to which component each subscale belongs. Energy / Fatigue, Pain, Health distress, Overall Quality of Life, Emotional well-being, Role limitation-emotional, Cognitive function belong to the 
Table 7: Factor loading (rotated) ${ }^{1}$ of two factor solution Component

\section{MSQoL-54 items}

Physical function

Health perceptions

Energy/fatigue

Role limitation-physical

Pain

Sexual function

Social function

Health distress

Overall quality of life

Emotional well-being

Role limitation-emotional

Cognitive function

Extraction method: Principal component analysis. 1: Rotation method: Varimax with kaiser normalization. The bold number in the table shows to which component each subscale belongs

Mental Health Composite because they have a greater score in this component. Physical function, Health perceptions, Role limitation-physical, Sexual function, Social function belong to the Physical Health Composite because they have a greater score in this component.

\section{Discussion}

An assessment of the quality of life of MS patients in addition to disease severity and disability level is important, because it provides unique information which would be beneficial for both, patients and clinicians.

We conducted this study to translate the 54-item Multiple Sclerosis Quality of Life questionnaire (MSQoL54 ) into Persian and then test its reliability and validity among Iranian MS patients. The same procedure has been done in Italian, ${ }^{[11]}$ French, ${ }^{[22,32]}$ French Canadian ${ }^{[33]}$ and Turkish ${ }^{[34]}$ languages. We fully adhered to forwardbackward translation, cognitive debriefing and cultural adaptation strategy to provide an applicable Persian version of the MSQoL-54 questionnaire. For this purpose we conducted a pilot study for the evaluation and reconstruction of our preliminary version.

The high percentage (37.5\%) of missing answers to the items of sexual function and satisfaction with sexual function is an intriguing feature. This was also reported in the United States survey, with 160 out of 179 (89\%) patients completing enough items on the sexual function scale to enable scoring and $19 \%$ missing data on Italian survey, ${ }^{[11]}$ with 150 out of 179 (84\%) answering the single item question on satisfaction with sexual function. Our finding is consistent with the results from the Italian and US surveys and also it could be attributable to the cultural characteristics and religious beliefs.

Reliability was assessed by Cronbach's alpha coefficient and it was acceptable for all subscales $(\alpha \geq 0.7)$ except for social function $(\alpha=0.654)$ and health perception $(\alpha=$
0.696). It might be due to cultural differences, because in Farsi language some of the questions in the two scales could be recognized as mental or physical problems and maybe some of the patients were unable to discriminate between them. In order to get a precise result, we suggest examining test re-test reliability further studies.

There were no studies on the reliability of MSQoL54 , therefore, we had no chance to compare our result with others.

The scale demonstrated convergent validity $(\alpha=0.962)$ and it is compatible with the study which assessed the validity of the MSQoL-54 in Italy. ${ }^{[11]}$

This study reports a high internal consistency of each scale regarding age, gender, marital status, education and duration of disease.

The results suggest a high internal consistency of 12 items of MSQoL-54 regarding EDSS and MS type. So, this questionnaire can be used for all MS patients with different EDSS and MS types.

In our study we found that the Physical Health $(P<0.001)$, Role limitation due to Physical Problems $(P=0.031)$, Energy $(P=0.004)$ and Health Perception $(P=0.029)$ scales have a strong association with marital status (single patients' scores were greater than married patients' ones). It might be due to this fact that married patients are concerned about being abandoned by their mates.

This study showed that Kurtzke's score (EDSS) was inversely correlated with Physical Health, Role limitation due to physical problems, Energy and Health Perception, Pain, Social Function, Cognitive Function, Health Distress, Overall Quality of Life and also Physical Health Composite and Mental Health Composite. This finding was confirmed by the Italian study. ${ }^{[11]}$

Age had a negative correlation with Physical Health, Role limitation due to physical problems, Energy and Health Perception, Pain, Social Function and Overall Quality of Life. Our finding is consistent with data from the Italian survey. ${ }^{[11]}$

In our study patients with higher fatigue scores and sleep scores had lower scores in all MSQoL-54 scales (both mental and physical health composite), but in contrast the Italian survey did not measure sleep and fatigue scales.

In this study Energy/Fatigue and Pain are categorized in the Mental Health Composite category. Because, in view of our patients these problems (or symptoms) are considered as mental problems instead of physical problems. This result could indicate that the surveyed patients misunderstood the words fatigue and energy, since these words in Farsi convey the meaning of distress and anxiety. We suggest that future studies investigate this item.

As some of the patients were not living in Shiraz we could not assess reliability by test-retest, but further studies should examine test-retest reliability in order 
to use this instrument on a large scale.

Our study has shown that the Persian version of MSQoL-54 questionnaire is easy to administer and is well accepted by patients. Neurological impairment, which was assessed by EDSS had an influence on the quality of life score but the reliability and validity of the Persian version of MSQoL-54 are satisfactory.

The study findings indicate that the Persian version of MSQoL-54 has a good structured characteristic and convergent validity, between items within scales and between scale correlations. Moreover, it is a reliable instrument that can be used for measuring the effects of MS on the Quality of Life.

In conclusion, we completed the translation, cultural adaptation, validation and reliability studies of MSQoL54 for Iranian patients. The Persian version of MSQoL-54 can be considered as a valuable and specific instrument to assess different aspects of HRQoL on MS patients and is applicable in clinical research and practice.

\section{Acknowledgement}

We are indebted to Dr. Barbara Vickrey for allowing us to adapt the original version of MSQoL-54, to Mr. Hamid Reza Pouremad, Dr. Shokrpour and Dr. Badiee, for help with the English and Mr. Sobhani, Mohammadi, Miss Khorrami, for helping us to test operators. This survey was supported by Grant No: 84-2526 of Research deputy of Shiraz University of Medical Sciences, Iran.

\section{References}

1. Wallin MT, Page WF, K urtzke J F. E pidemiology of multiple sclerosis in US veterans VIII. L ong-term survival after onset of multiple sclerosis. B rain 2000;123:1677-87.

2. B eatus J, O'N iel J, Townsend T, Robrecht $K$. The effect of a one-week retreat on self-esteem, quality of life and functional ability for persons with multiple sclerosis. N eurology Report, Sep 2002.

3. Spilker B, editor. Quality of life and pharmacoeconomics in clinical trials, $2^{\text {nd }}$ ed. L ippincott-Raven: P hiladelphia; 1994.

4. Patrick DL, D eyo RA. Generic and disease-spesific measures in assessing health status and quality of life. Med Care 1989;27:S217-32.

5. Mitenburger $\mathrm{C}, \mathrm{K}$ obelt $\mathrm{G}$. Quality of life and cost of multiple sclerosis. Clin N eurol N eurosurg 2002;104:272-5.

6. Lintern TC, B eaumont J G, K enealy PM, Murrell RC. Quality of L ife $(\mathrm{Q} O \mathrm{~L})$ in severely disabled multiple sclerosis patients: comparison of three QoL measures using multidimensional scaling. Qual L ife Res 2001;10:371-8.

7. Kurtzke J F. Rating neurologic impairment in multiple sclerosis: An expanded disability status scale (E DSS). N eurology 1983;33:1444-52.

8. N ortvedt MW, Riise T, Myhr K M, N yland H I. Quality of life in multiple sclerosis: measuring the disease effects more broadly. Neurology 1999;53:1098-103.

9. J anardhan $V, B$ akshi R. Quality of $L$ ife and its relation to brain lesions and atrophy on magnetic resonance images in 60 patients with multiple sclerosis. Arch N eurol 2000;57:1485-91.

10. P fennings L E, Cohen L, Van der Ploeg H M. Assessing the quality of life of patients with multiple sclerosis. In: Multiple scler osis: Clinical challenges and controversies. Thompson AJ , Polman C, H ohlfeld R, editors. Martin Duntiz: L ondon; 1997. p. 195-210.

11. Solari A, Filippini G, Mendozzi L, Ghezzi A, Cifani S, B arbier E, \& al. Validation of I talian multiple sclerosis quality of life 54 questionnaire. J N eurol N eurosurg Psychiatry 1999;67:158-62.
12. Rothwell PM. Quality of life in multiple sclerosis. J Neurol Neurosurg Psychiatry 1998;65:433.

13. Sadovnick AD, E bers GC. E pidemiology of multiple sclerosis: A critical overview. Can J Neurol Sci 1993;20:17-29.

14. M CH orney CA, Ware J E J r, L u J F, Sherbourne CD. The MOS 36-item Short-Form H ealth Survey (SF-36): III. Tests of data quality, scaling assumptions, and reliability across diverse patient groups. Med Care 1994;32:40-66.

15. Vickrey B G, H ays RD, H aronir NI, Myers LW, Ellison GW. A healthrelated quality of life measure for multiple sclerosis. Qual Life Res 1995;4:187-206.

16. B runet DG, Hopman WM, Singer MA, Edgar CM, Mackenzie TA M easurement of health-related quality of life in multiple sclerosis patients. Can J Neurol Sci 1996;23:99-103.

17. L ankhost GJ , J elles F, Smiths RC, Polman CH, Kuik DJ , Pfennings $L E$, \& al. Quality of life in multiple sclerosis: The disability and impact profile(DIP). J N eurol 1996;243:469-74.

18. Cella DF, Dineen MA, Arnason B, Reder A, Webster KA, K arabatsos G, $\notin$ al. Validation of functional assessment of multiple sclerosis quality of life instrument. N eurology 1996,47:129-39.

19. Aronson KJ . Quality of life among persons with multiple sclerosis and their caregivers. N eurology 1997;48:74-80.

20. Acquadro C, L afortune L, M ear I. Quality of life in multiple sclerosis: Translation in French Canadian of the MSQ LL -54. Health Qual L ife Outcomes 2003;1:70.

21. Yamamoto T, Ogata K, Katagishi M, Shimizu H, Ogawa M, Yamamura $T$, $₫$ al. Validation of the J apanese-translated version M ultiple Sclerosis Quality of L ife-54 instrument. Rinsho Shinkeigaku 2004;44:417-21.

22. Acquadro $C$, J ambon $B, E$ llis $D, M$ arquis $P$. L anguage and translation issues. In Quality of life and P harmacoeconomics clinical trials. Speaker B, editor. L ippencourt- Raven Publishers: P hiladelphia; 1996. p. 575-85.

23. Ware J E J r, Sherbourne CD. The MOS 36-item short-form health survey (SF-36). I. Conceptual framework and item selection. Med Care 1992;30:473-83.

24. Solari A. Role of health-related quality of life measures in the routine care of people with multiple sclerosis. Health Qual L ife Outcomes 2005;3:16.

25. B uysse DJ , Reynolds CF $3^{\text {rd }}$, M onk TH, B erman SR, Kupfer DJ. The pittsburgh sleep quality index: A new instrument for psychiatric practice and research. Psychiatry Res 1989;28:193-213.

26. Mear I. D ifficulties of international clinical trials: Cultural adaptation of quality of life questionnaires. In: $\mathrm{H}$ ealth-related quality of life and Patientreported outcomes: Scientific and useful outcome criteria. Chassany 0 , Caulin C, editors. Springer Verlag Publishers: Paris; 2002. p. 55-62.

27. Fayers PM, Machin D. Quality of life assessment, analysis and interpretation. J ohn Wiley and Sons: N ew York; 2000. p. 289-306.

28. Poser CM , B rinar VV. Diagnosis criteria for multiplesclerosis. Clin N eurol N eurosurg 2001;103:1-11.

29. M CH orney CA, Ware J E, Raczek AE. The MOS 36 item short form health survey (SF-36) II. Psychometric and clinical tests of validity in measuring physical and mental health constructs. Med Care 1993;31:247-63.

30. Cronbach LJ. Coefficient $\alpha$ and the internal structure of tests. Psicometrika 1951;16:297-334.

31. B land J M, Altman D G. Survival probabilities (theK aplan-M eier method). BMJ 1998;317:1572.

32. L eplege A, E cosse E, Verdier A, Perneger T. The French SF-36 health survey: translation, cultural adaptation and preliminary psychometric evaluation. J Clin E pidemiol 1998;51:1013-23.

33. Vernay D, Gerbaud L, B iolay S, Coste J, Debourse , Aufauvre D, \& al. Quality of life and multiple sclerosis: Validation of the French version of the self-questionnaire (SE P-59). Rev N eurol 2000;156:247-63.

34. I diman E, U zunel F, Ozakbas S, Yozbatiran N, Oguz M, Callioglu B , e al. Cross-cultural adaptation and validation of multiple scler osis quality of life questionnaire (MSQ oL -54) in a Turkish multiple sclerosis sample. J Neurol Sci 2006;240:77-80.

Accepted on 11-06-2007

Source of Support: Grant No: 84-2526 of Research deputy of Shiraz University of Medical Sciences, Iran., Conflict of Interest: None declared. 